\title{
Incorporating environmental degradation in closed form adhesive joint stress analyses.
}

A D Crocombe, Faculty of Engineering and Physical Sciences, University of Surrey, UK

Running title: Closed form durability stress analyses

\begin{abstract}
A review of the literature reveals that although the effects of environmental degradation have been incorporated into some recent finite element stress analyses of adhesive joints, closed form solutions have not yet been developed. Due to its ease of use, such a closed form approach would be very useful for screening analyses of potential joint configurations. Two different closed form approaches have been developed. Both use simple, yet demonstrably reasonable, assumptions about the affect of moisture on the mechanical properties of the adhesive and both use a Fickian diffusion model to determine moisture distribution in the joint. The simplest approach is based on limit state assumptions and results in a simple nondimensionalised equation relating residual strength to the time of exposure. The equation is in the form of an infinite series and is also presented as a design chart for even easier assess. The second approach is the adaptation of a generalised non-linear adhesive sandwich analysis. The advantage of this is that it is applicable to a wide range of bonded joints and not just the single lap joint in the closed form solution. Further it is not limited by limit state assumptions and gives the adhesive peel and shear stress distribution for any loading condition and exposure time. Both approaches were illustrated by the application to a typical single lap joint, exposed at $85 \%$ RH for 230 days.
\end{abstract}

Key words

durability modelling, closed form bonded joint analysis, material degradation, limit state, global yielding, bonded joint design tool, general joint analysis

\section{Introduction}

One of the major challenges facing the adhesive technologist is ensuring joint integrity in the presence of a wet environment. Moisture penetrates the joint and can degrade both the cohesive 
properties of the adhesive and the interface between the adhesive and substrate. Watts and coworkers [1-3] and others have undertaken excellent work investigating the nature of this degradation at a molecular level using surface science techniques. Clearly it is important to be able to use this and other information when designing adhesively bonded structures to ensure their fitness for purpose. One way of validating a bonded joint design is through long term exposure and testing. This is being undertaken [4-5] but does carry significant time penalties. In an attempt to overcome this problem researchers have used use more aggressive environments to accelerate the degradation [6]. However there is always the concern that a degradation mechanism will be induced in this accelerated testing that does not occur in service conditions.

An alternative approach is to develop predictive modelling techniques that simulate the response of a bonded structure to service conditions. Recently, significant advances have been made in this area [7,8] with coupled mechanical-diffusion finite element analyses that can incorporate hygro-thermal residual as well as mechanical stresses together with progressive damage modelling controlled by environmental dependent damage parameters. Such analyses are extremely computationally intensive and require considerable expertise to implement. In order to allow for a more rapid, preliminary screening of candidate bonded joints it would be useful to have an analysis tool that is more readily accessible. Analyses where the governing equations have been formulated specifically for adhesive joints can provide this sort of ease of solution and accessibility. Such analyses are commonly termed "closed form" analyses in comparison with the more open or generalised nature of finite element analyses.

There is a long history of closed form adhesive joint analyses commonly believed to start with the shear lag approach outlined by Volkersen [9]. A detailed account of these analyses can be found elsewhere [10] and only a brief summary is given here. Volkersen's analysis neglects peel stresses and hence is more applicable to a double lap joint. A characteristic of this and all subsequent analyses is the peaking of the adhesive stresses at the overlap ends where the load transfer from one substrate through the adhesive to the other substrate occurs. Goland and Reissner [11] included substrate bending in their analysis of the single lap joint and this enabled expressions to be developed for adhesive peel as well as shear stresses. Also an expression was developed that gave the substrate bending moment and consequently the shear force in terms of the applied axial load. Following this, Volkersen [12] extended their original analysis to include the adhesive peel stresses. Unlike the outer substrates the centre substrate experienced no bending. 
Renton and Vinson [13] made the next significant contribution to the analysis of single lap joints, including the effect of shearing in orthotropic substrates. The maximum peel and shear stresses are somewhat lower than those found by Goland and Reissner [11]. The adhesive shear and peel stress were still assumed constant across the thickness of the adhesive. Ojalvo and Eidinoff [14] incorporated a linear variation of shear strain across the adhesive thickness but neglected substrate shearing. This allowed the adhesive shear stress to become zero at the free surface at the overlap ends. Delale et al [15] included adhesive longitudinal stress, in addition to the shear and peel stresses, however these adhesive stresses were assumed constant across the adhesive thickness. Yang and Pang [16] use a Fourier series approach to model unbalanced joints with three adhesive stress components included. A different way of modelling substrate shear was introduced by Tsai et al [17], but only the adhesive shear stress was included. Sawa et al [18] modelled an unbalanced single lap joint by considering the two substrates and the adhesive layer as three separate strips, subjected to common but unknown traction along the interfaces and appropriate tractions on the other faces.

A number of authors including Allman [19] and Adams and Mallick [20] have adopted a different approach to obtain a global stress analysis of adhesive joints applying the variational principle of complementary energy. There is generally good correlation between these analyses and the more conventional closed form solutions.

To a certain extent these refinements in the distribution of stress across the adhesive thickness are of more theoretical than practical interest. The reason for this is that an exact solution for the linear elastic stresses in an adhesive joint [21] show that the stresses are infinite due to the bi-material singularities that occur at the overlap ends. In the context of bonded joint design there are various ways of dealing with this singularity that include a) defining the singularity strength and intensity (much like conventional fracture mechanics) and b) processing the stress or strain field over a finite zone surrounding the singular point [22]. One very common approach is to average the adhesive stresses across the adhesive layer and this, in essence, is what the early closed form analyses do.

All the analyses discussed above assume linear behaviour of the adhesive and substrate. Many modern adhesive systems exhibit significant non-linearity in their stress strain behaviour and hence analyses based on linear material behaviour may be of limited use for strength 
assessment. To overcome this Hart-Smith [23, 24] extended double and single lap joint analyses modelling the adhesive as an elasto-plastic material. The analyses were simplified by uncoupling the shear and peel stresses, assuming elasto-plastic behaviour for the former and elastic for the latter. By assuming that joint failure occurs at a critical level of plastic strain, Hart-Smith develops design charts for predicted joint strength.

The solutions above have been derived for specific joint configurations (ie single and double lap joints) and thus are of limited use for application to a wider range of joint configurations. Bigwood and Crocombe [25,26] generalised the solution of Goland and Reissner [11] for an arbitrarily end loaded overlap thus widening its range of applicability considerably, as illustrated in Fig 1. They incorporated a full non-linear representation of the adhesive layer where both the peel and shear stresses contribute to adhesive yield. Good correlation was found between their analyses and non-linear FE solutions. This was extended further [27] to include plastic deformation in the substrates as well as the adhesive. It should be pointed out that the substrate moment, shear and axial loads are required at the overlap ends. These end loads may be found directly or, for more complex configurations, from an analysis of the structure containing the joint. In these structural analyses it is not necessary to model the joint in detail.

Weitsman [28] and others (eg [20]) have included the swelling effect of hygrothermal strains in lap joint analysis as an initial strain but do not model the degradation of material properties that is induced by the moisture. It can be seen that the basic closed form approach has been developed to accommodate non-linear material behaviour of arbitrary joint configurations. However, these analyses cannot currently accommodate any form of material environmental degradation and this is the purpose of the work discussed in this paper. Two separate approaches have been outlined. In the following section an estimate for the reduced strength of a joint exposed to environmental degradation has been derived based on a limit state assumption. This results in a simple equation and a chart for the upper bound of the failure load of a degraded single or double lap joint. This is followed by a section that outlines the extension of the non-linear arbitrarily end loaded overlap closed form analysis of Bigwood and Crocombe [26] to accommodate moisture dependent material parameters. This is second approach is applicable to a wide range of adhesive joints, as illustrated in Fig 1.

\section{Limit state solution for environmentally degraded adhesive single lap joints}


A limit state solution gives an upper bound to joint strength, the failure load being found by assuming that all the adhesive reaches its maximum load carrying capacity before joint failure occurs. Crocombe [29] termed this global yielding and showed that it was applicable to a wide range of lap joints. Such joints should be able to reach yield over most of the adhesive layer before local failure of the adhesive at the overlap ends occur. This obviously depends on the ductility of the adhesive and the overlap length. Global yielding can be applied to most conventional single and double lap joints constructed with modern adhesives, but will be less applicable as the overlap length increases.

In this solution a limit state load is found for a 2D representation of a joint following exposure to a known environment for a known period of time. It has been assumed that the transport of the moisture into the joint is governed by Fickian diffusion and thus from standard texts such as Crank [30] it can be shown that the moisture concentration $(c)$ at a certain distance from the overlap end (x) within an initially dry $(c=0)$ joint of overlap length $L$ at a time $(t)$ is given by Eqn 1, where $c_{o}$ is the saturation moisture uptake of the adhesive and $D$ is the diffusion coefficient.

$$
c(x, t)=c_{o}-\sum_{0}^{\infty} \frac{4 c_{o}}{(2 j+1) \pi} \sin \left[\frac{(2 j+1) \pi x}{L}\right] e^{\frac{-(2 j+1)^{2} D \pi^{2} t}{L^{2}}}
$$

This is illustrated schematically in Fig 2

The constitutive response of adhesives is known to be a function of moisture content. Both the modulus and ultimate strength reduce with increasing moisture content. Data measured within the authors group [31-33] for four structural adhesives are shown in Table 1. It can be seen that the degree of degradation varies and that there appears to be a correlation with the maximum water uptake. Data for one of these adhesive systems (B) are shown in more detail in Fig 3 and this has formed that basis for modelling later in this paper. It can be seen that the reduction in both properties with moisture is essentially linear and the analysis developed in this section makes use of this observation. 
From basic equilibrium the limit state load $\left(P_{\max }\right)$ carried by a single lap joint can be obtained by integrating the maximum adhesive shear stress $\left(\tau_{\max }\right)$ along the overlap length $(L)$. As the maximum (ultimate) shear stress reduces with increasing moisture content the maximum shear stress attainable by the adhesive is higher in the (drier) centre of the overlap than the (wetter) outer region. This is illustrated schematically in Fig 4 and Eqn 2

$$
P_{\max }=\int_{0}^{L} \tau_{\max }\left(c / c_{o}\right) d x
$$

By assuming that the maximum shear stress $\left(\tau_{\max }\right)$ varies linearly with the moisture $(c)$ from a value of $\tau_{d r y}$ when $c=0$ to a value of $\tau_{\text {wet }}$ when $c=c_{o}$ the maximum shear stress can be expressed as

$$
\frac{\tau_{\max }}{\tau_{d r y}}=1-\frac{c}{c_{o}}\left(1-\frac{\tau_{w e t}}{\tau_{d r y}}\right)
$$

Substituting Eqns [3] into [2] and manipulating gives

$$
\begin{aligned}
& P_{\max }=\tau_{d r y}\left(L-F_{\tau} \int_{0}^{L} \frac{c}{c_{o}} d x\right) \\
& \text { where } F_{\tau}=1-\frac{\tau_{w e t}}{\tau_{d r y}}
\end{aligned}
$$

Substituting Eqn [1] into [4] and manipulating gives

$$
P_{\max }=\tau_{d r y}\left(L\left(1-F_{\tau}\right)+F_{\tau} \sum_{0}^{\infty} \frac{4}{(2 j+1) \pi} e^{-(2 j+1)^{2}\left(\frac{D \pi^{2} t}{L^{2}}\right)} \int_{0}^{L} \sin (2 j+1) \frac{\pi x}{L} d x\right)
$$

Evaluating the integral in Eqn [5] and simplifying further finally gives:

$$
\frac{P_{\max }}{\tau_{d r y} L}=\left[1-F_{\tau}\left(1-\frac{8}{\pi^{2}} \sum_{0}^{\infty} \frac{1}{(2 j+1)^{2}} e^{-(2 j+1)^{2}\left(\frac{D \pi^{2} t}{L^{2}}\right)}\right)\right]
$$


The parameter $F_{\tau}$ represents the degree of degradation that the adhesive experiences on exposure to moisture. When $F_{\tau}=0$ there is no degradation in the adhesive on saturation whilst $F_{\tau}=1$ indicates that the adhesive loses all its strength on exposure to moisture. For the adhesives shown in Table $1 \mathrm{~F}_{\tau}$ varies between 0.53 and 0.91 . This equation enables the limit state load to be determined for any adhesive system characterised by its dry and saturated ultimate stresses, the diffusion coefficient, the time of exposure and the overlap length.

To facilitate the application of this limit state solution Eqn 6 is presented graphically for a number of $F_{\tau}$ values in Fig 5. This enables the limit state load to be readily assessed without evaluation of Eqn 4. Further some important trends can be noted. As expected the curves become asymptotic to $\tau_{\mathrm{w}} / \tau_{\mathrm{d}}\left(=1-F_{\tau}\right)$ at long exposure times. Under these conditions the entire overlap is saturated and is thus uniformly, fully degradaded. At short exposure times the limit state load approaches the fully dry limit state load. At intermediate exposure time the limit state load reduce gradually from the fully dry value to the fully degradaded value.

\section{Development of the Generalised Adhesive Sandwich (GAS) analysis to incorporate environmental degradation.}

\subsection{Derivation and solution procedure}

The original non-linear GAS analysis, as presented by Bigwood and Crocombe (1992), required the solution of the following six non-linear differential equations 


$$
\begin{aligned}
& \frac{d T_{1}}{d x}=\frac{E_{s} \gamma_{x y}}{2\left(1+v_{p}\right)} \\
& \frac{d V_{1}}{d x}=\frac{E_{s} \varepsilon_{y}}{\left(1+v_{p}^{2}\right)} \\
& \frac{d M_{1}}{d x}=V_{1}-\frac{\left(h_{1}+t_{a}\right) E_{s} \gamma_{x y}}{4\left(1+v_{p}\right)} \\
& \frac{d \varepsilon_{y}}{d x}=\kappa \\
& \frac{d \kappa}{d x}=f_{1}\left(M_{1}, T_{1}, x\right) \\
& \frac{d \gamma_{x y}}{d x}=f_{2}\left(M_{1}, T_{1}, x\right)
\end{aligned}
$$

Further details are given in the original paper [26]. In these equations $T_{1}, V_{1}$ and $M_{1}$ are the tension, shear and moment in the upper substrate at a distance $\mathrm{x}$ from the overlap end, see Fig 1 and $\mathrm{h} 1$ and ta repreent the thickenss of the upper substrate and the adhesive layer respectively. The parameters $\gamma_{x y}$ and $\varepsilon_{y}$ represent the shear and peel strains in the adhesive corresponding the adhesive shear and peel stresses, $\sigma_{y}$ and $\tau_{x y}$ respectively. Adhesive nonlinearity is modelled using a deformation theory of plasticity, that links the total stress to the total strain using the secant modulus $E_{s}$ and a plastic Poisson's ratio $v_{p}$ The secant modulus is defined in terms of the von Mises equivalent adhesive stress $\left(\sigma_{e q}\right)$ and strain $\left(\varepsilon_{e q}\right)$ as illustrated in Fig 6 and the plastic Poisson's ratio is defined in Eqn 8, where $E$ and $v$ are the elastic modulus and Poisson's ratio respectively.

$$
v_{p}=\frac{1}{2}\left[1-\frac{E_{s}}{E}(1-2 v)\right]
$$

Normally only the adhesive strains (and associated stresses) are required but the upper substrate loads are obtained from the solution and these can be used to find upper substrate stresses and also from equilibrium, lower substrate loads and stresses if required. These equations are solved using a finite difference boundary value procedure. A direct method rather than a shooting method has been used as the full set of boundary conditions at $x=0$ are not known and the shooting method was found to have convergence problems. The boundary conditions for this problem are the upper substrate tension, shear and moment at each end of the overlap. 
The original solution uses a single hyperbolic tangent model to define the generic stress-strain response of the adhesive

$$
\sigma_{e q}=\operatorname{Atanh}\left[\frac{E \varepsilon_{e q}}{A}\right]
$$

All the parameters have been defined above apart from $A$, which is the ultimate adhesive equivalent stress.

To extend this analysis to include degradation due to moisture it is necessary to replace the single adhesive material model with an infinitely varying material model which is moisture dependent. The solution is then carried out in two steps. The initial step determines the moisture distribution in the adhesive, by the application of Eqn 1. The diffusion coefficient D and the saturation moisture uptake are commonly found from gravimetric tests. There is aso a considerable amount of published data available for different adhesives. The second step is the stress analysis and here the actual material curve being used at any given point in the adhesive depends upon the moisture and thus the position in the overlap. This is illustrated schematically in Fig 7, which shows the lowest (most degraded) stress strain curve occurring at the overlap ends where the adhesive is wettest and the highest (least degraded) curve occurring at the centre of the overlap where the adhesive is driest.

Such a moisture dependent material model can be implemented by making the parameters $A$ (ultimate stress) and $E$ (modulus) in Eqn 9 moisture dependent. Typical variation of modulus and ultimate stress with moisture has already been shown in Fig 3, where it was noticed that both appeared to decrease linearly with increasing moisture content. Thus the model has been extended by rewriting Eqn 10 as

$$
\sigma_{e q}=A_{c} \tanh \left[\frac{E_{c} \varepsilon_{e q}}{A}\right]
$$

defining $A_{c}$ and $E_{c}$ as: 


$$
\begin{aligned}
& \frac{A_{c}}{A_{d r y}}=1-\frac{c}{c_{o}}\left(1-\frac{A_{w e t}}{A_{d r y}}\right) \\
& \frac{E_{c}}{E_{d r y}}=1-\frac{c}{c_{o}}\left(1-\frac{E_{w e t}}{E_{d r y}}\right)
\end{aligned}
$$

where subscripts dry and wet refer to the properties at fully dry and fully saturated conditions respectively and $c$ and $c_{0}$ refer to moisture levels as discussed in Eqn 1.

The solution procedure is summarised in the flow chart shown in Fig 8. In addition to geometry, loads and substrate material, also required are the dry and wet modulus and ultimate stress values, the diffusion coefficient and time of exposure, all for the environment under consideration.

The moisture distribution is calculated analytically using Eqn 1 at a number of points along the adhesive layer. Values of the moisture at positions between these points are found using interpolation. It has been found that only a few terms of the infinite series in Eqn 1 are required to obtain convergence.

An initial finite difference (FD) mesh having $n$ - 1 equally spaced internal points (and hence $n$ regions) is defined for the solution of the six first order non-linear differential equations presented in Eqn 7. This mesh may be refined (discussed later) if convergence of the non-linear solution process cannot be achieved on this initial mesh. To start the solution an initial guess for values of all six of the unknowns at all $n+1$ mesh points are required. Linear interpolation can be used for the substrate loads $\left(T_{1}, V_{l}\right.$ and $\left.M_{l}\right)$ as boundary values of these are given at each end of the overlap. It has been found that assuming a value of zero for the other 3 variables $\left(\varepsilon_{y}\right.$, $\kappa$ and $\left.\gamma_{\mathrm{xy}}\right)$ at all points provides an acceptable starting point for the solution.

Each of the 6 differential equations are written as $1^{\text {st }}$ order difference equations over each of the $n$ regions. This provides $6 n$ equations in the $6(n+1)$ unknowns. The remaining 6 equations come from the 6 boundary condition values (the 3 substrate loads at each end of the joint). The equations can be expressed in matrix form

$$
[\mathrm{K}][\mathrm{y}]-[\mathrm{R}]=[\mathrm{F}]=0
$$


where $[K]$ is the $6(n+1)$ square matrix of coefficients of the unknowns stored in the $6(n+1)$ column vector $[y]$. The $6(n+1)$ column vector $[R]$ contains the RHS of each of the equations. The $6(n+1)$ column matrix $[F]$ is known as the residual and should be zero when the unknowns (held in $[y]$ ) take their correct values.

In a linear problem Eqn 13 can be solved directly to give the unknowns. However in a nonlinear problem such as this, the equations must be solved iteratively. Here a Newton-Raphson approach was adopted. This involved finding the residual and the Jacobian of the residual and using the two to find an improved solution. Both the residual and the Jacobian require the secant modulus $\left(E_{s}\right)$ and the plastic Poisson's ratio $\left(v_{p}\right)$. Finding these values involves the use of the moisture dependent hyperbolic tangent model evaluated at the appropriate moisture level. The terms $E_{s}$ and $v_{p}$ are coupled and hence at each material point an iterative loop is used to find converged values corresponding to the adhesive strains at that point ( $\varepsilon_{y}$ and $\left.\gamma_{x y}\right)$.

Convergence for such non-linear problems can be difficult and various techniques have been incorporated to enhance the convergence. It is not appropriate to go into detail here but just to indicate that these techniques include ramping in the non-linearity (by incrementing the load) and refining the FD mesh where large errors are identified. The errors are assessed using higher order terms in the FD expansion.

\section{$\underline{3.2 \text { Validating analyses }}$}

To validate the implementation and illustrate the effectiveness of this solution procedure a set of stress analyses have been undertaken on a typical single lap joint. It should be emphasised that unlike the limit state solution outlined in the previous section this analysis is applicable to any joint geometry that contains an end loaded substrate-adhesive sandwich.

\section{Configurations analysed}

The single lap joint analysed had $1 \mathrm{~mm}$ aluminium substrates, $0.25 \mathrm{~mm}$ adhesive layer and $12.5 \mathrm{~mm}$ overlap and was subjected to an axial load of $400 \mathrm{~N} / \mathrm{mm}$. This joint configuration is 
shown in Fig 9a and the corresponding end loaded overlap region is shown in Fig 9b. The Goland and Reissner (1945) bending moment factor was used to calculate the corresponding shear and moment loads on the overlap ends.

Three different conditions have been assumed: dry linear material, dry non-linear material and exposed in $85 \% \mathrm{RH}$ for 230 days non-linear material. The diffusion coefficient and dry and saturated adhesive modulus $(E)$ and flow stress $(A)$ values are given in Table 1 . The data used are representative of typical structural epoxy adhesives. The moisture distribution in the joint after 230 days at $85 \% \mathrm{RH}$ is shown in Fig 10. It can be seen that even after such a relatively long exposure period the centre of the joint is reasonably dry. In practice diffusion would also occur in the adhesive in the out of plane direction. However as a typical single lap joint width is $25 \mathrm{~mm}$ (twice the overlap length) the moisture distribution is dominated by diffusion in the plane.

For clarity the stress strain curves obtained using the material parameters in Table 1 are shown in Fig 11. Again it should be emphasized that these are representative properties. It can be seen that both the modulus and the ultimate stress are strongly dependent on the level of moisture in the adhesive. The lowering of the modulus would tend to reduce and spread the adhesive stress and strain state whilst the lowering of the ultimate stress will reduce the load carrying capacity of the joint.

\section{$\underline{\text { Results and discussion }}$}

The resulting shear stress and shear strain distributions are shown in Fig 12a and b respectively. The strains from the analysis with linear material behaviour are the lowest as in the other analyses the adhesive becomes more flexible and hence, for a given load the deflection and thus the strains will increase. The stress distribution for this linear analysis matches the shape of the strain distribution, which is to be expected from a linear analyses as the modulus is constant.

Including adhesive plasticity (non-linear) results in higher strains. The strain distribution also becomes more peaky, this is because yielding spreads in from both ends of the overlap and so the edges are relatively more compliant than the centre resulting in a more rapid increase of strains in these outer regions. Consideration of the shear stresses show the yielding that occurs 
when non-linearity is included. It would appear that, for the level of load considered, the adhesive has yielded over the first few $\mathrm{mm}$ at each end of the overlap. Yielding occurs as a result of the shear and peel stresses, which combine to give an equivalent stress. The reason that the shear stresses dip down towards the ends is because in these regions the adhesive peel stress (not shown here) rises quite sharply and the combined equivalent stress cannot exceed the dry ultimate stress of $69.3 \mathrm{MPa}$.

Strains from the full moisture dependent (wet) analysis are even higher. This is because, in addition to dry yielding, the moisture also reduces the linear modulus and the ultimate stress even further, increasing the flexibility of the adhesive further. The stresses show an interesting trend. The dips at the overlap ends are not only caused by the sharply rising peel stresses contributing to the combined equivalent stresses. The moisture in this end region also reduces the ultimate stress that can be carried. Unlike the dry non-linear stresses the stresses do not dip towards the centre of the joint, even though the shear strains do. This is because of the moisture profile, shown in Fig 10. The adhesive becomes drier as the centre of the overlap is approached. Thus at the centre the modulus and ultimate stress are higher and hence the adhesive can transmit more stresses even at reduced levels of strain. This higher stress occurring at the centre of the joint is a feature that has been noted elsewhere [34].

An indication of how close the "wet" analysis was to limit state conditions can be found by using Fig 5 to evaluate the limit state load. With the diffusion coefficient of $2.42 \times 10^{-13} \mathrm{~m}^{2} / \mathrm{s}$, a time of exposure of 230 days and on overlap length of $12.5 \mathrm{~mm}$ the abscissa of the graph can be evaluated to be 0.304 . Referring to Fig 5 it can be seen that although this is a relatively low abscissa value a reasonable degree of degradation has occurred. This is entirely consistent with the moisture profile shown in Fig 10. Table 1 shows that the wet to dry ultimate stress ratio $\left(\tau_{\mathrm{w}} / \tau_{\mathrm{d}}\right)$ is 0.72 , which corresponds to an $\mathrm{F}_{\tau}$ value of 0.28 The ordinate corresponding to the intersection of this curve and the abscissa value of 0.304 is 0.89 . Assuming von Mises yielding the dry shear strength corresponding to an ultimate stress of $69.3 \mathrm{MPa}$ is $40 \mathrm{MPa}$. From this and the overlap length the limit state load can be evaluated to be $445 \mathrm{~N} / \mathrm{mm}$. As expected, this is a little higher than the load applied in the analysis, which was $400 \mathrm{~N} / \mathrm{mm}$. This implies that the "wet" analysis results shown in Fig 12 are reasonably close to the limit state condition. 


\section{Conclusions}

A brief review of existing closed form adhesive joint analyses has revealed many developments on the original analyses of Goland and Reissner. However many of these cannot incorporate non-linear material behaviour because of the complexity of their formulations.

Environmental degradation has only been incorporated in stress analyses of joint using complex finite element solutions. No record has been found of any closed form analysis that has been developed to incorporate the degrading effects of moisture on the adhesive material properties.

In this paper two approaches have been outlined for incorporating moisture degradation into closed form solutions. The first is a limit state approach that will be more applicable for shorter overlap or ductile adhesives. Non-dimensionalised results are presented in equation and graphical form that are immediately accessible to other workers.

An approach that is not restricted by the limit state conditions was then outlined. This consisted of adapting and existing non-linear solution to incorporate moisture transport and moisture dependent material properties. This can be used to give the adhesive stress and strain distribution throughout the joint for any given loading condition.

Due to the wide range of joint configurations and the non-linear and moisture dependent material models this is probably the most advanced closed form solution available for adhesively bonded joints.

This approach currently only incorporates the degradation of the cohesive adhesive properties and thus is really applicable to those joints where the degraded adhesive forms the weak link. The degradation of the interface between the adhesive and the substrate can be incorporated indirectly by a reduction in the adjacent adhesive material properties. However, in principle it is possible to incorporate the interface directly using fracture mechanics and this is an area for further development. Another area that needs consideration is the effect of swelling strains due to the absorbed moisture in the adhesive, on the stress distribution within the joint. It should be relatively straightforward to include this effect in the closed form analysis.

\section{Acknowledgements}


The author would like to thank Mr Stephen Lilley who helped code the moisture dependent analyses.

\section{References}

[1] Watts J.F. and Castle J.E., J. Mater. Sci. 19, 2259-2272 (1984).

[2] Davis S.J. and Watts J.F, J. Mater. Chem. 6, 479-493 (1996).

[3] Korenburg C.F., Kinloch A.J. and Watts J.F., J. Adhesion 80, 169-201 (2004).

[4] Parker BM, Vide Les Couches Minces 50-272, 590-593 (1994)

[5] Ashcroft, I.A., Digby, R.P., Shaw, S.J., J. Adhesion, 75, 175-202 (2001).

[6] Hamade RF, Dillard DA, J Adhes Sci And Tech, 17-9, 1235-1264 (2003)

[7] Crocombe A.D., Hua Y.X., Loh W.K., Wahab M.A. and Ashcroft I.A., Int. J. Adhesion Adhesives 26, 325-336 (2006).

[8] Liljedahl C.D.M., Crocombe A.D., Wahab M.A. and Ashcroft I.A., J. Adhesion Sci. Technol. 19, 525-547 (2005).

[9] Volkersen O, Luftfahrtforschung, 15, 41-47 (1938)

[10] Crocombe AD, Stress Analysis, in Adhesive Bonding Science Technology and Applications, ed R D Adams, , (Woodhead Publishing Ltd, Cambridge 2005) Chap. 5, pp. 91-122

[11] Goland M and Reissner E, J Applied Mechanics, 66, 17-27 (1945)

[12] Volkersen O, Construction Metallique, 4, 3 (1965)

[13] Renton WJ and Vinson JR, J Applied Mechanics, 101-106 (1977)

[14] Ojalvo U and Eidinoff HL, AIAA, 16-3, 204-211 (1978)

[15] Delale F, Erdogan F and Aydinoglu MN, J Composite Materials, 15, 249-271 (1981)

[16] Yang C and Pang SS, J Eng Matls and Tech, 118-2, 247-255 (1996)

[17] Tsai MY, Oplinger DW and Morton J, Int J Solids and Structures, 35-12, 1163-1185 (1998)

[18] Sawa T and Suga HJ, Adhes Sci and Tech, 10-12, 1255-1271 (1996)

[19] Allman DJ, Quart J Mech and Appl Math, 30, 377-386 (1977)

[20] Adams RD and Mallick V, J Adhesion, 38, 199-217 (1992)

[21] Bogy DB, J Applied Mechanics, 35, 460-466 (1968)

[22] Groth HL, Int J Adhesion and Adhesives, 8-2, 107-113 (1988)

[23] Hart-Smith LJ, NASA report CR112235, Langley Research Centre (1973)

[24] Hart-Smith LJ, NASA report CR112236, Langley Research Centre (1973) 
[25] Bigwood DA and Crocombe AD, Intl J Adhesion and Adhesives, 9, 229-242 (1989)

[26] Bigwood DA and Crocombe AD, Int J Adhesion and Adhesives, 10-1, 31-41 (1990)

[27] Crocombe AD and Bigwood DA, J Strain Anal for Mech Des, 27-4, 211-218 (1992)

[28] Weitsman Y, J Comp Mat, 11, 378-394 (1977)

[29] Crocombe AD, Int J Adhesion and Adhesives, 9-3, 145-153 (1989)

[30] Crank J, The Mathematics of Diffusion (Oxford Science Publications 1979), $2^{\text {nd }}$ Ed,

[31] Hambly HO, The Strength of Adhesive Bonded Joints Degraded by Moisture, PhD thesis, University of Surrey (1998)

[32] Loh WK, Modelling Interfacial Degradation in Adhesively Bonded Structures, PhD thesis, University of Surrey, 2002

[33] Liljedahl CDM, Modelling the Interfacial Degradation in Adhesively Bonded Joints, PhD thesis, University of Surrey, 2006

[34] Crocombe AD, Int J Adhesion and Adhesives, 17-3, 229-238 (1997) 

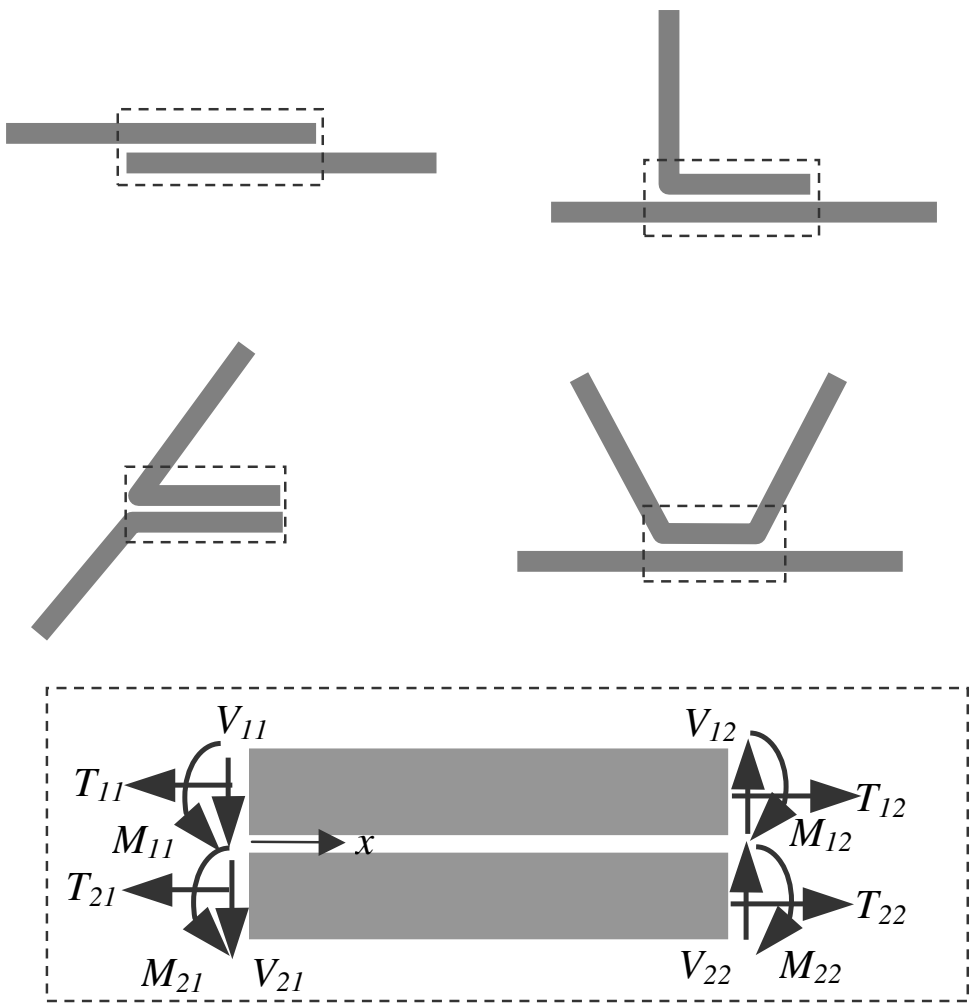

Fig 1 -A selection of potential joint configurations all of which contain an end loaded overlap.

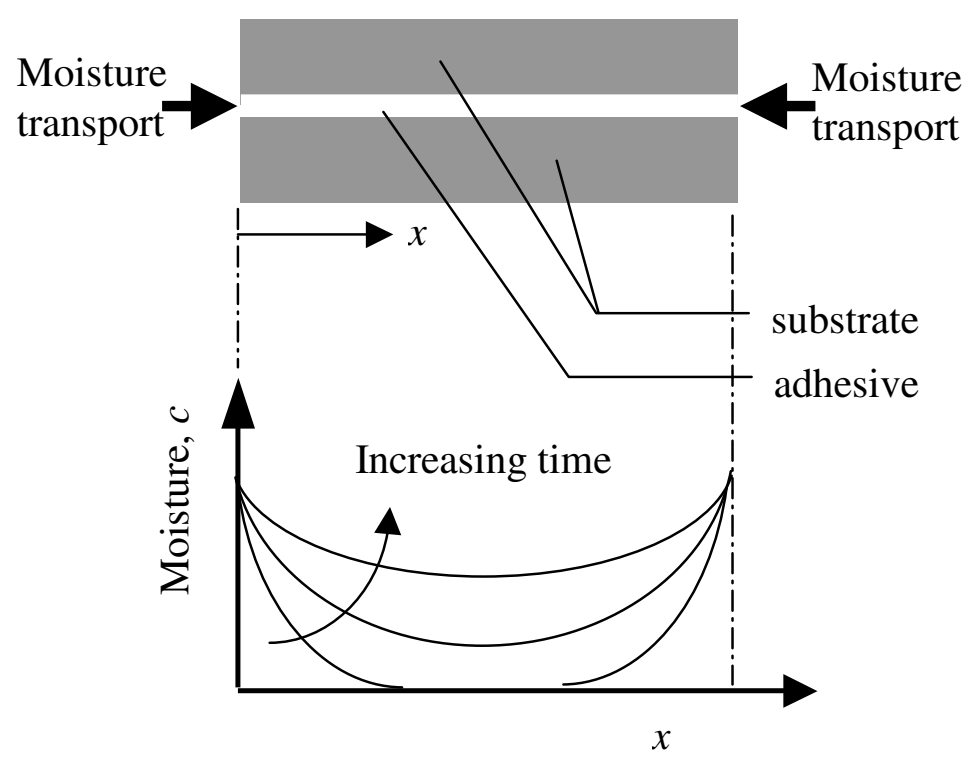

Fig 2 - Moisture distribution within the adhesive joint 


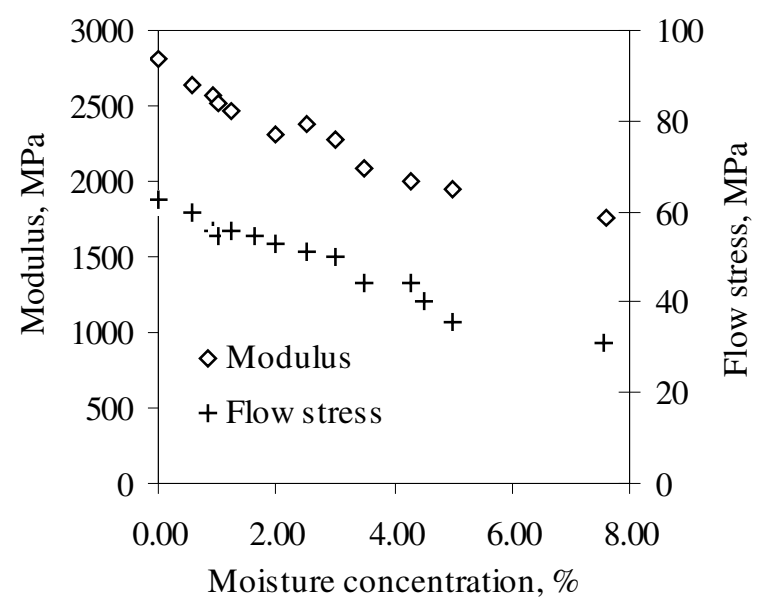

Fig 3 - Variation of modulus and ultimate strength of a toughened epoxy with moisture

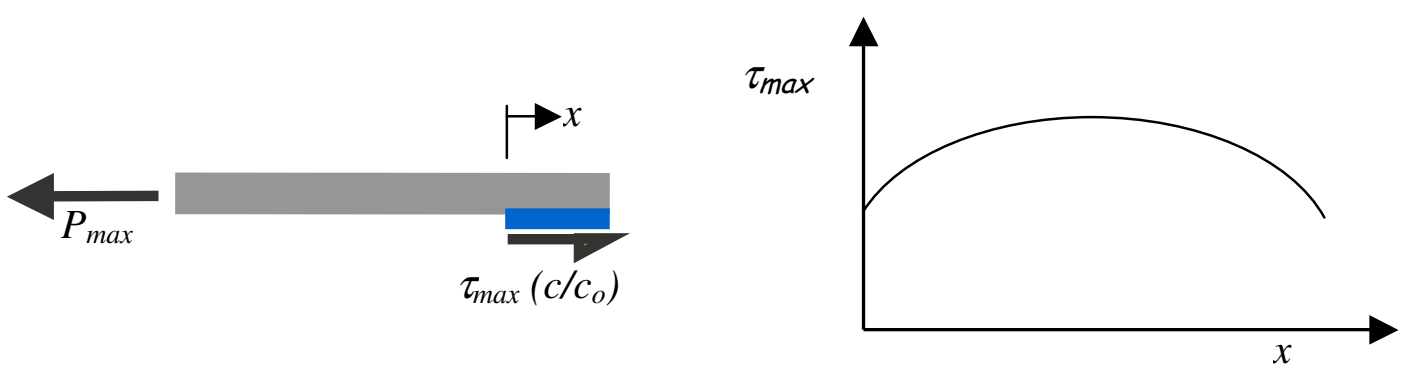

Fig 4 - Schematic illustration of limit state load evaluation. 


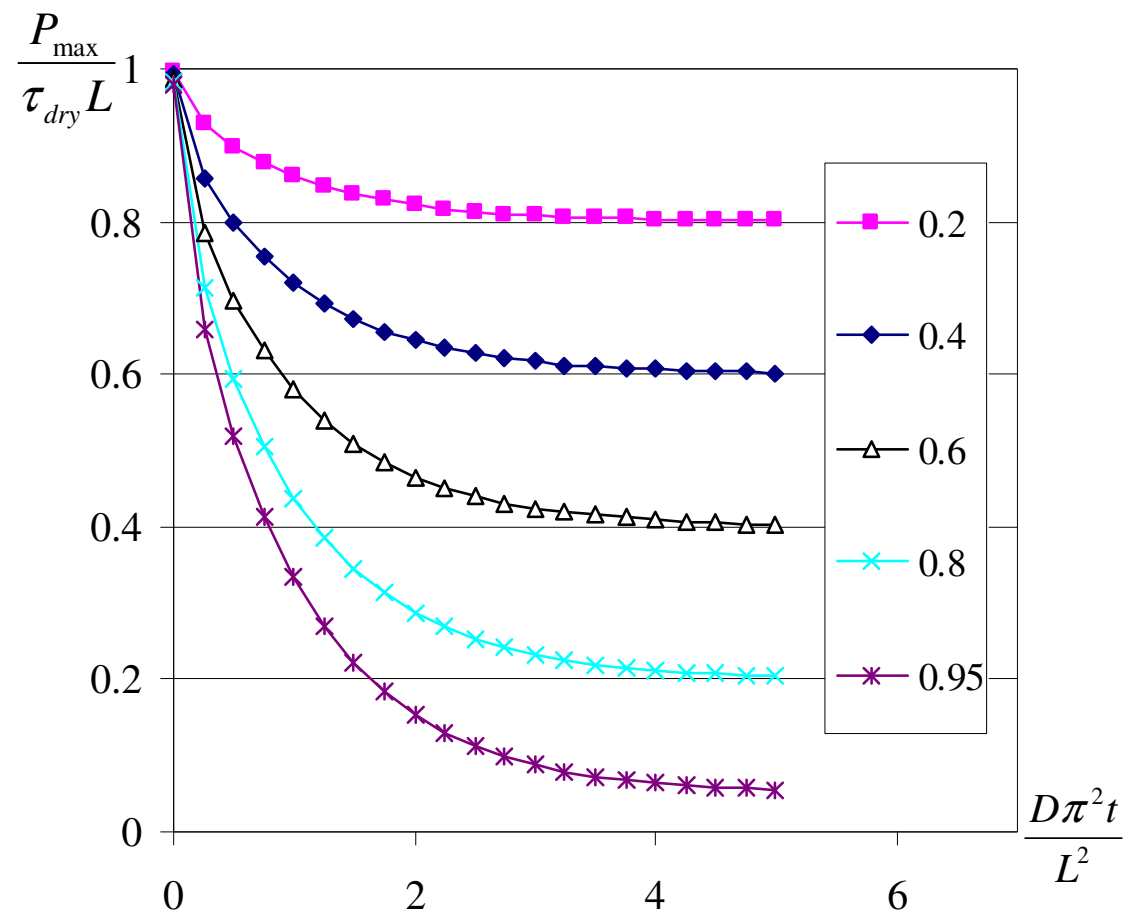

Fig 5 - Showing the variation of the normalised limit state load with non-dimensionalised time of exposure for a range of $F_{\tau}$ values.

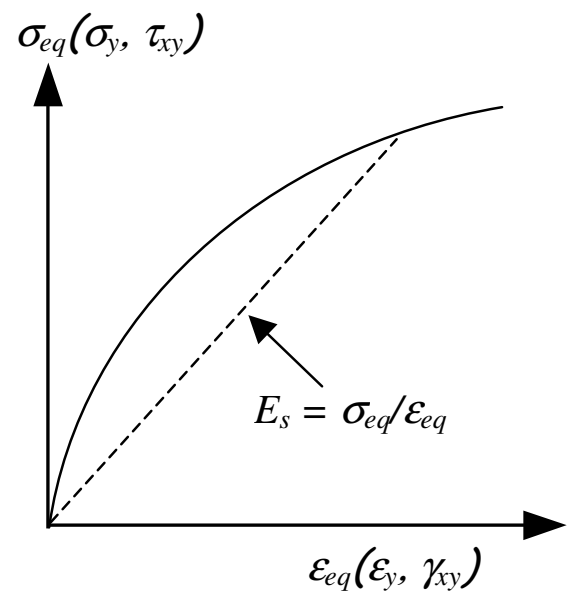

Fig 6 - Non-linear adhesive stress-strain curve. 


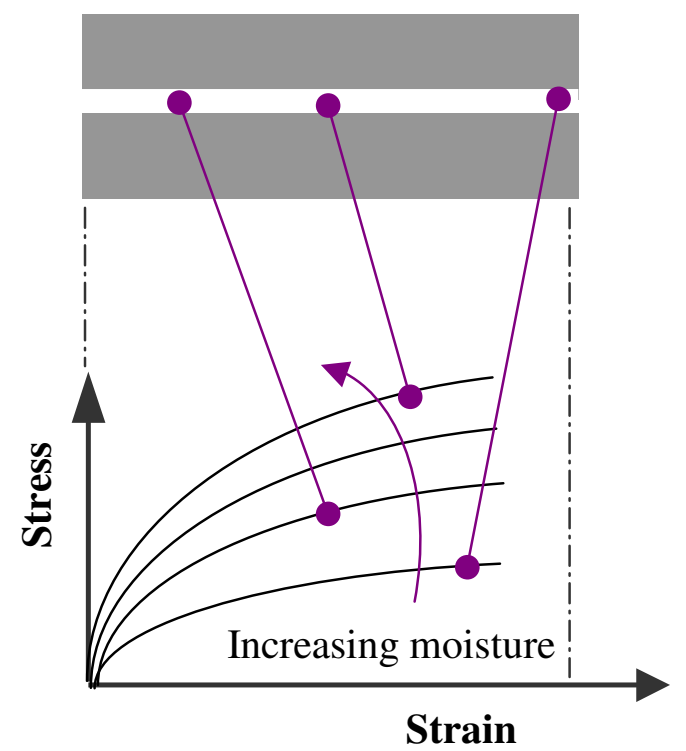

Fig 7 - Schematic illustration of the moisture dependent adhesive stress strain response.

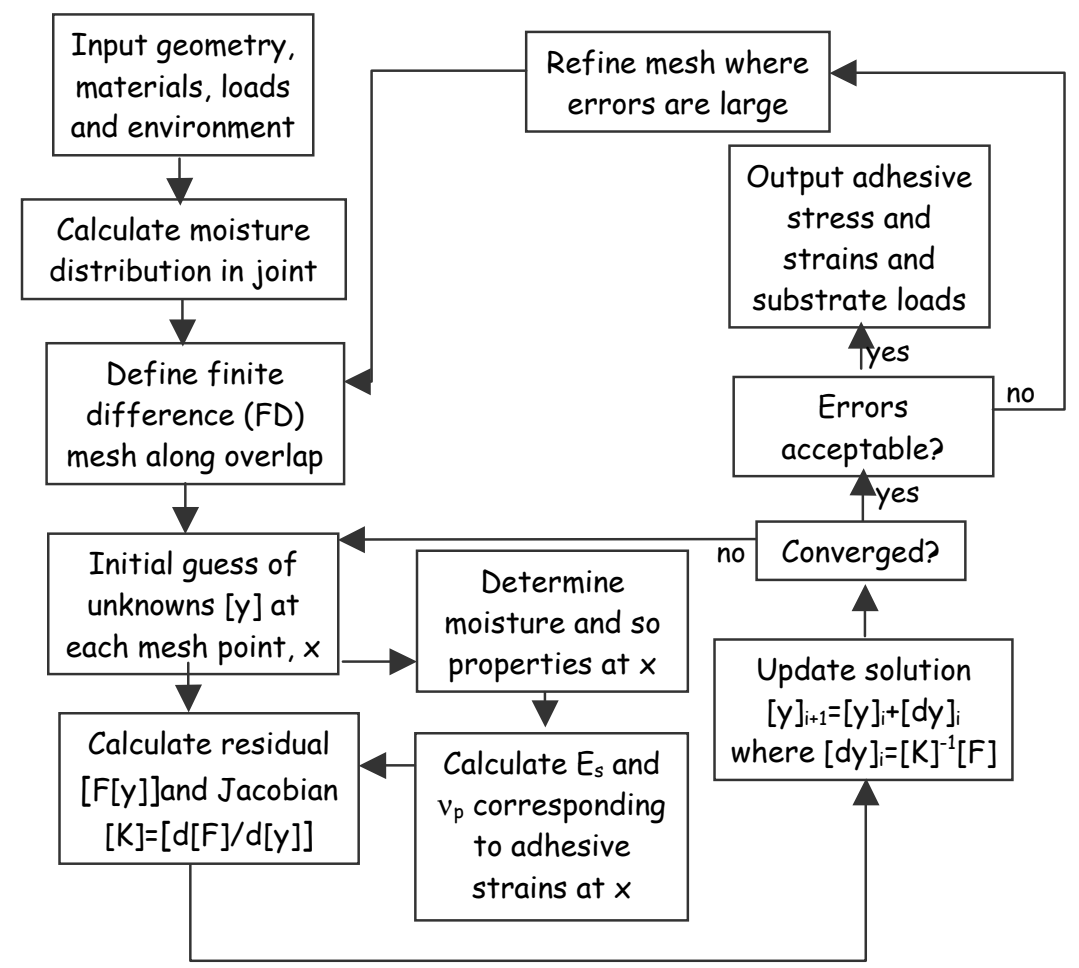

Fig 8 - Flow chart of the modified GAS solution procedure 


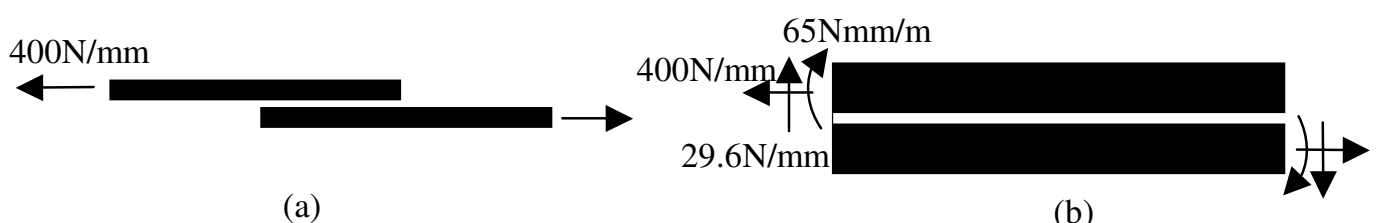

(a)

(b)

Fig 9 - Single lap joint configuration and end load conditions

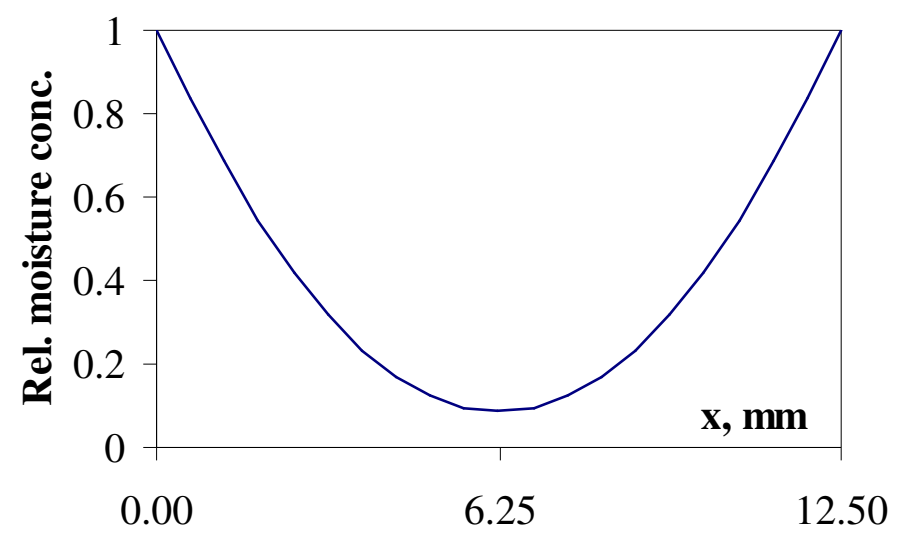

Fig 10 - Moisture distribution along the overlap after exposure in $85 \%$ RH after 230 days. 


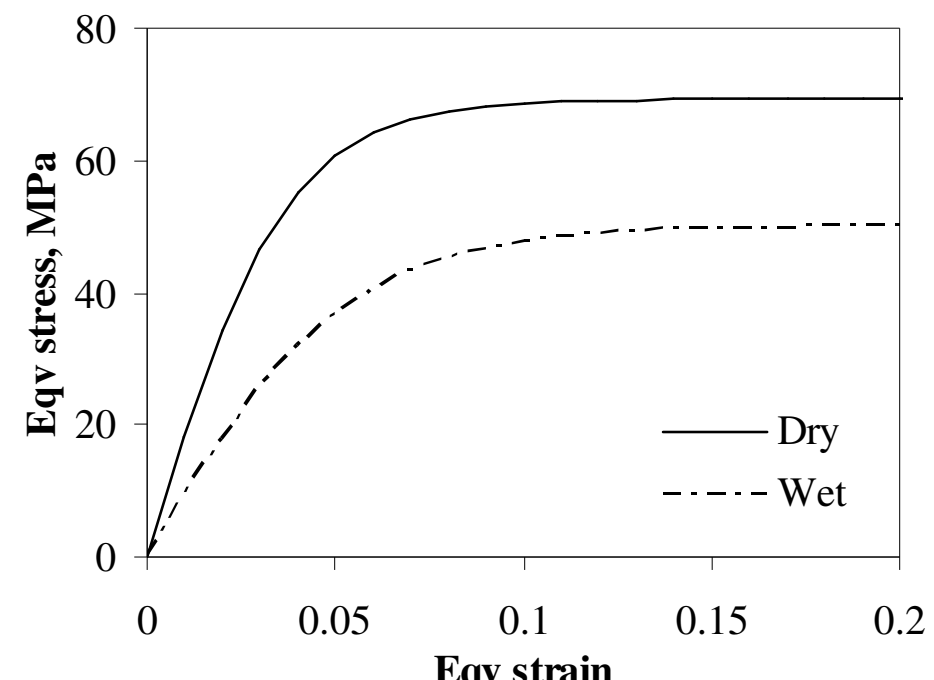

Fig 11 - Dry and saturated bulk adhesive tensile stress strain curves

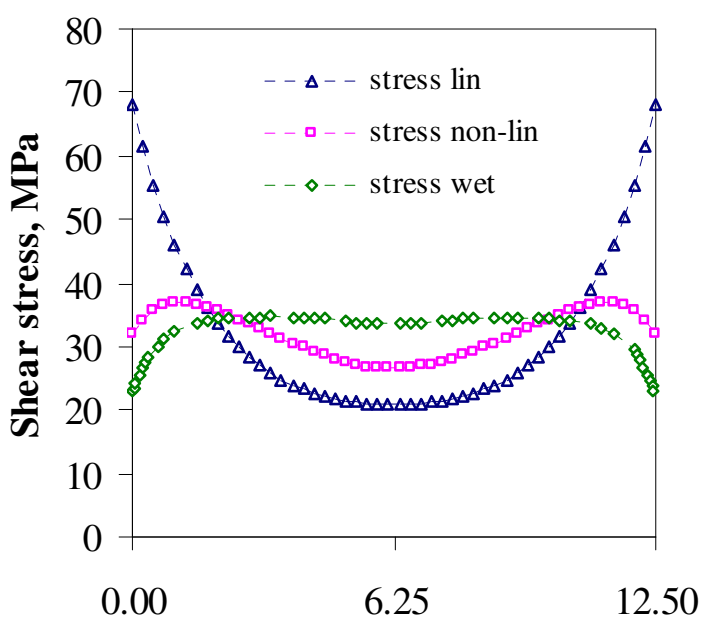

Dist along overlap, $\mathrm{mm}$

(b)

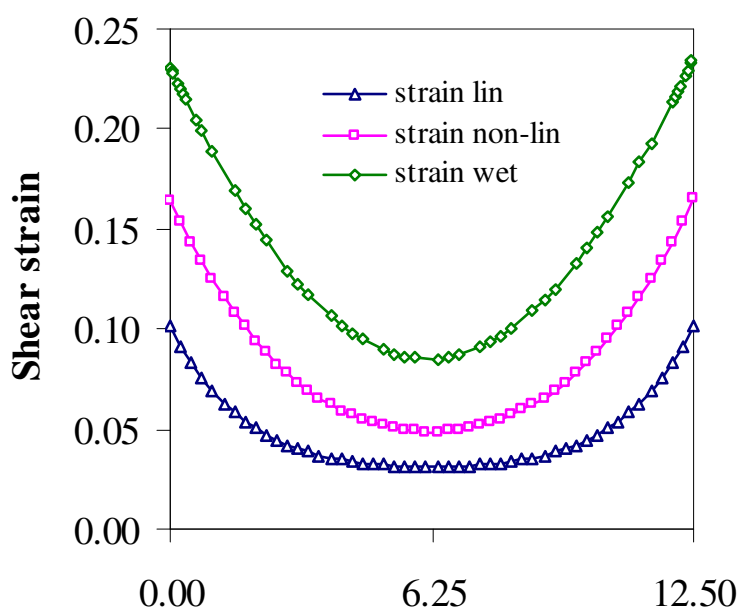

Dist along overlap, mm

(a)

Fig 12 - Comparison of stress and strain distributions 
Table 1 - Moisture degraded modulus and strength values for four different structural adhesives

\begin{tabular}{l|l|l|l|l|l}
\hline Adhesive & $\begin{array}{l}\text { Saturated } \\
\text { moisture uptake }\end{array}$ & $\begin{array}{l}\text { Dry } \\
\text { modulus }\end{array}$ & $\begin{array}{l}\text { Saturated } \\
\text { modulus }\end{array}$ & $\begin{array}{l}\text { Dry } \\
\text { strength }\end{array}$ & $\begin{array}{l}\text { Saturated } \\
\text { strength }\end{array}$ \\
\hline A & $9.0 \%$ & $2000 \mathrm{MPa}$ & $340 \mathrm{MPa}$ & $32 \mathrm{MPa}$ & $13 \mathrm{MPa}$ \\
B & $7.6 \%$ & $2800 \mathrm{MPa}$ & $1800 \mathrm{MPa}$ & $65 \mathrm{MPa}$ & $30 \mathrm{MPa}$ \\
$\mathrm{C}$ & $3.9 \%$ & $2900 \mathrm{MPa}$ & $2500 \mathrm{MPa}$ & $53 \mathrm{MPa}$ & $48 \mathrm{MPa}$ \\
$\mathrm{D}$ & $2.0 \%$ & $2000 \mathrm{MPa}$ & $1500 \mathrm{MPa}$ & $45 \mathrm{MPa}$ & $32 \mathrm{MPa}$ \\
\hline
\end{tabular}

